\title{
Some visual influences on human postural equilibrium: Binocular versus monocular fixation
}

\author{
CHARLES R. FOX \\ Washington University School of Medicine, St. Louis, Missouri
}

\begin{abstract}
The importance of vision for postural equilibrium has long been known; traditionally, this visual contribution to the control of posture has been analyzed primarily in terms of optical and retinal phenomena. Recently, however, there has been some suggestion that binocular and monocular fixation of identical stimuli have differential effects. Three experiments were conducted in order to measure self-generated movement (sway during quiet standing) of the body's center of gravity while field structure, ankle proprioception, and binocular/monocular fixation were varied. Field structure was varied from total darkness, to the presence of single and multiple LEDs in the dark, to full field structure (i.e., the richness of the feedback information was varied). Ankle proprioception was varied by changing foot position from side-by-side to heel-to-toe positions. Results indicate that (1) ankle-joint input is a significant factor in reducing sway, (2) binocular fixation attenuates sway relative to monocular fixation, under otherwise identical visual conditions, and (3) this difference persists in total darkness. Taken together, the data indicate that the visual influence on postural equilibrium results from a complex synergy that receives multimodal inputs. A simple optical/retinal explanation is not sufficient.
\end{abstract}

Many sources of information could, in principle, be used for postural control; however, clinical and empirical evidence has demonstrated that vision plays a major role.

Visual motion cues are an important source of information for visual stabilization of posture. Observers exposed to large, moving visual fields show disturbances of posture, equilibrium, and self-motion perception; these disturbances seem related to parameters of the visual motion (Dichgans \& Brandt, 1978; Dichgans, Held, Young, \& Brandt, 1978; Dichgans, Mauritz, Allum, \& Brandt, 1975; Lee \& Lishman, 1975, 1977; Lestienne, Soechting, \& Berthoz, 1977; Lishman \& Lee, 1973). The relation between posture and visual motion is further supported by studies in which motion velocity cues have been minimized through the use of stroboscopic illumination or visual "stabilization." This minimization resulted in postural disequilibrium when touch-pressure-kinesthetic (TPK) inputs generated by the reaction forces of the feet against the support surface were altered (Amblard,

Parts of this research were submitted, as part of a doctoral dissertation, to the Graduate Faculty of Brandeis University; the remainder was completed during postdoctoral training at Columbia University. I wish to thank James R. Lackner and Len Matin, who supervised my doctoral and postdoctoral training, respectively. Both have been very helpful during the research and writing. I also wish to thank Sheldon Ebenholtz, Herschel Leibowitz, and G. Timothy Petito, who have read and discussed earlier versions of this paper. Support for the research and for preparation of the manuscript was provided by Grants EY05929, AG06442, and T32 EY07057 from the NIH, Grant BNS 8617059 from the NSF, Fight For Sight award PD 85-060 in memory of Hermann M. Burian, and a grant from the Veterans Administration Medical Center of New York. Correspondence should be addressed to Charles R. Fox, Department of Otolaryngology, Washington University School of Medicine, Box 8115, 517 S. Euclid Ave., St. Louis, MO 63110.
Carblanc, \& Cremieux, 1980; Amblard \& Cremieux, 1976; Diener, Dichgans, Guschlbauer, \& Bacher, 1986; Paulus, Straube, \& Brandt, 1984).

This "visual driving" of sway may be related to the phenomena of visually perceived self-motion perception (vection). Studies in which seated subjects have estimated the magnitude of horizontal and vertical vection have yielded results similar to results that suggest a relation of body pitch to a linearly moving visual surround (see Figure 1 in Berthoz, Lacour, Soechting, \& Vidal, 1979). The quantitative similarity between estimates of the gain and phase of visually driven postural sway and vection suggests that visual influences on postural sway can be profitably explored through the use of more general models of visual spatial orientation.

All such models must deal with the same problem. Since the optic flow at the eye is influenced by object motions, eye movements, and body movements, the visual influence on postural equilibrium is similar to the general problem of perception of a stable world. That is, how do observers dissociate and respond appropriately to each source of motion information represented in the optical transformation? Postural equilibrium requires the maintenance of the appropriate relation between the body's center of gravity and the base of support; therefore, only movements of the body are relevant. To maintain equilibrium, the observer must be able to dissociate the causes of the visual transform and respond to the appropriate aspect. The problem of factoring retinal motion into its components is not new; two general classes of theory address this issue: comparator theory and visual flow theory.

Comparator theory (see Figure 1) suggests that retinal change is evaluated against independent kinesthetic infor- 


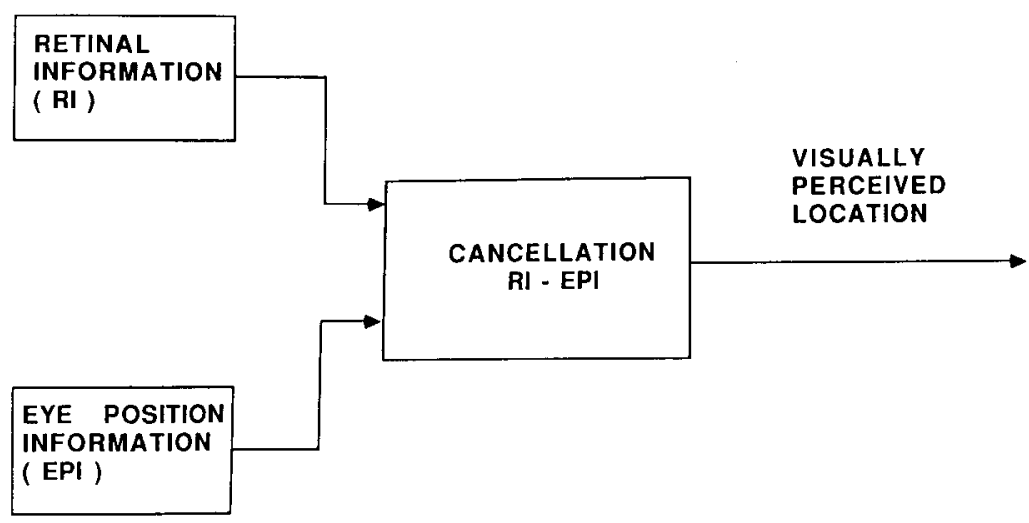

Figure 1. Diagrammatic representation of a standard comparator mechanism as traditionally conceived.

mation. An extraretinal signal encoding the magnitude of the eye movement is said to "cancel" a retinal signal encoding the magnitude of retinal image motion (Helmholtz, 1866/1962; Holst \& Mittelstaedt, 1950; Matin, 1982; Wertheim \& Bles, 1984; White, Post, \& Leibowitz, 1980). Visual flow theory has been primarily concerned with the optic flow as a source of independent information that would allow factoring of the retinal pattern (Gibson, 1950, 1966, 1979; Johansonn, 1976; Lee \& Lishman, 1977). In brief, flow theory suggests that different physical situations give rise to different types of retinal pattern transformations. Figure 2 illustrates these relations.

Neither theory as it currently exists is sufficient to explain the data on visual control of postural equilibrium; the sources of information implicated by both theories are present and useful in a normal environment (Matin \& Fox, 1989). To date, however, comparator theory has not per- mitted specific predictions, and so, this class of theory, although it should not be rejected, cannot be directly tested. Visual flow theory, on the other hand, does allow specific predictions regarding self-motion perception.

Given the relation between vection and postural equilibrium, it is reasonable to include predictions about postural equilibrium by expanding flow theory as follows: Visual flow theory is based on decomposition of the optical pattern at the eye. Given a rigid environment, this pattern is a vector field that contains a "propriospecific" component (Gibson, 1966), which is determined through the observer's position, orientation, and movement, relative to the environment. One example of a propriospecific pattern is motion parallax, in which the registered differential movement of targets in depth provides information indicating self-motion and body sway. In principle, the visual information available in these propriospecific pat-

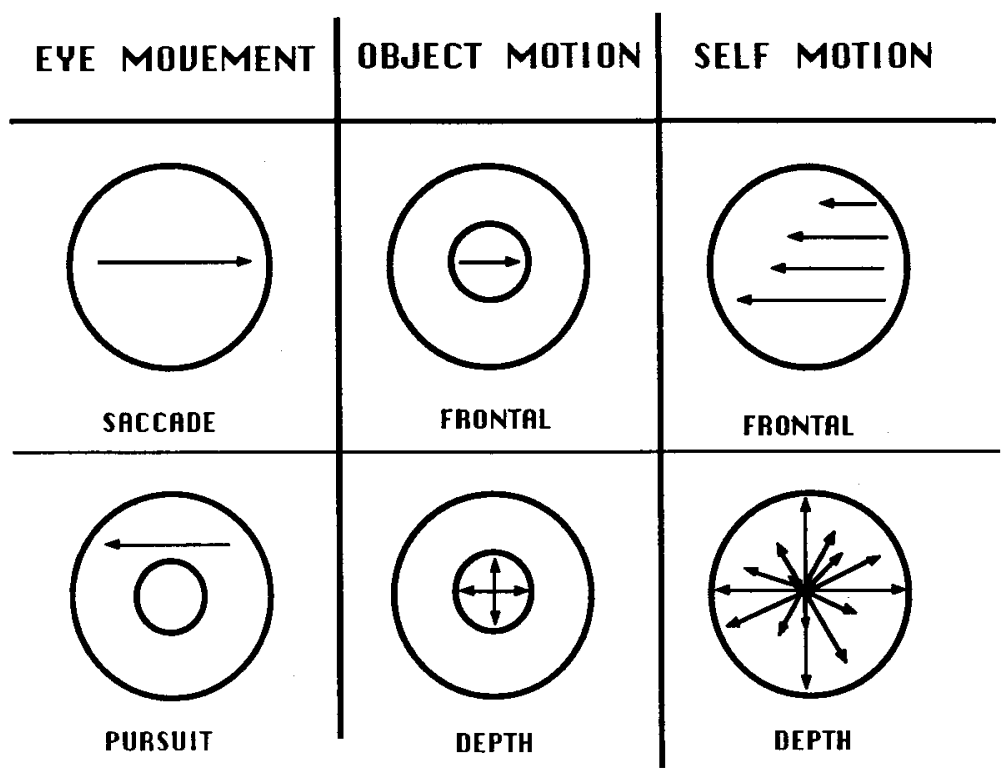

Figure 2. Diagrammatic representation of the motion vector fields resulting from different physical situations. Large circles represent the visual fields, small circles represent objects in the field, and arrows represent the motion vector. 
terns is available to both binocular and monocular vision. However, our pilot observations suggested that there are binocular-monocular differences in the ability of identical stimulus conditions to stabilize sway. Such differences have never been reported, and so they are systematically investigated here for the first time.

\section{EXPERIMENT 1}

\section{Method}

Data collection and analysis were in accord with standards recommended by the International Society of Posturography (see Kapteyn et al., 1983).

Subjects. Ten subjects between the ages of 18 and 40 received payment for their voluntary participation. The subjects were screened for self-reported abnormalities relevant to postural equilibrium and vision. All subjects had normal or corrected-tonormal vision and reported general good health.

Stimulus display. A movable table had three upright bars, each with a crossbar; the bars were painted flat black. The structure, though rigid, could be moved relative to the subject. It had six light- emitting diodes arranged as in Figure 3. Bar 1 (closest to the observer) was $145 \mathrm{~cm}$ from the observer's eye; each light's intensity was set at $0.5 \log \mathrm{fL}$.

Six stimulus conditions were used (see Figure 4); in all the conditions except full, the experimental room was in total darkness, the only illumination being the specified target lights: ${ }^{1}$

1. Eyes closed (EC): No target lights were illuminated, and the subjects closed their eyes. This was the baseline.

2. Full illumination (full): The experimental room was illuminated by two $100-\mathrm{W}$ incandescent bulbs (ambient illumination of $1.6 \log \mathrm{fc}$ ); all six target lights were on, and the normal laboratory environment provided full field structure.

3. Single target (T): A single target light provided a fixation point but no configural information about body sway.

4. Motion parallax (MP): Three target lights aligned in depth made available potential information maximally relevant to left-right $(L / R)$ body sway.

5. Expansion-contraction (Exp/Con): Three target lights aligned in the frontoparallel plane made available potential information maximally relevant to fore-aft (F/A) body sway.

6. Expansion-contraction and motion parallax (Exp/Con-MP): Three target lights aligned as a diagonal in depth made available

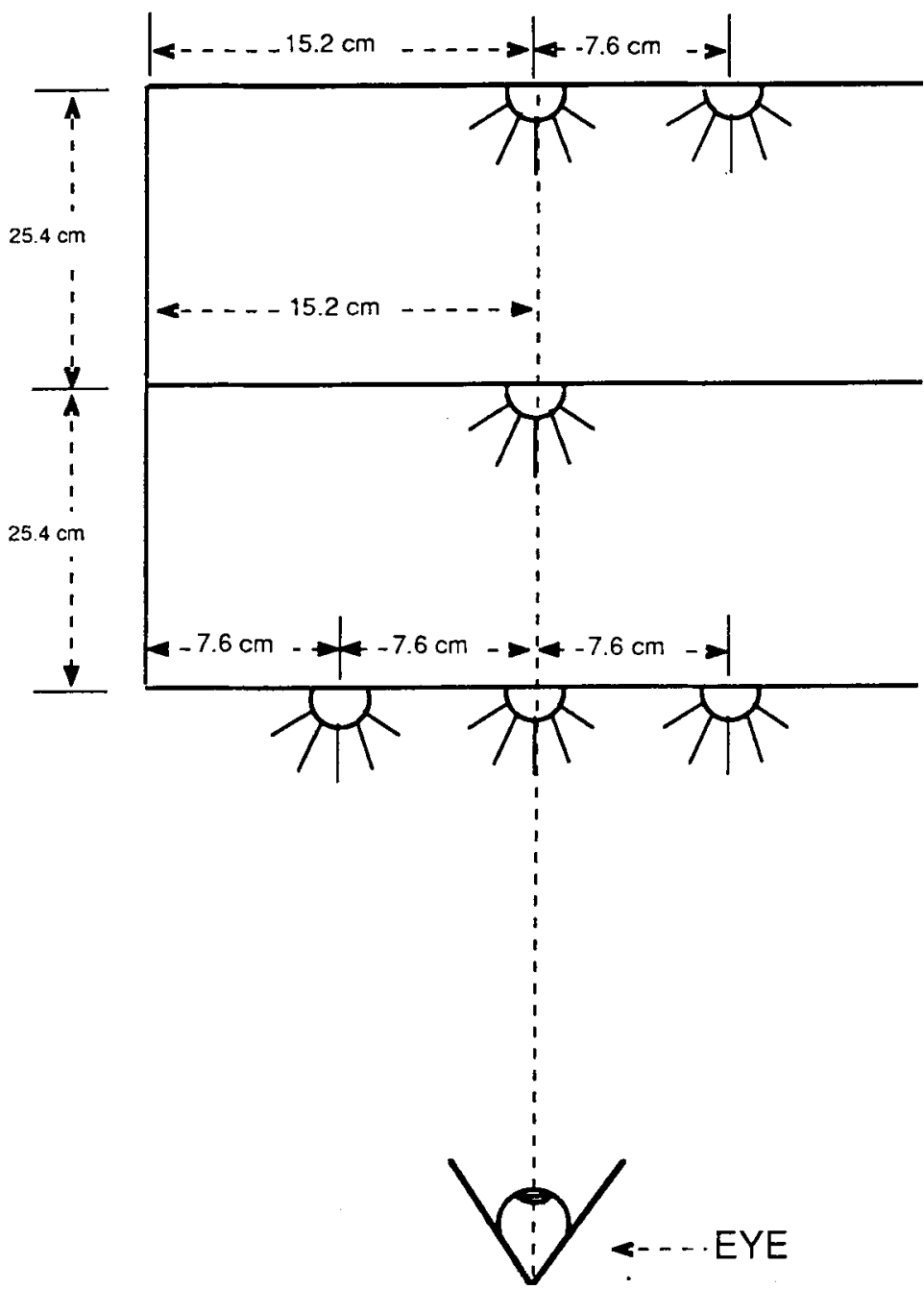

Figure 3. Diagrammatic representation of the LED array used in the experiments, viewed from above. 


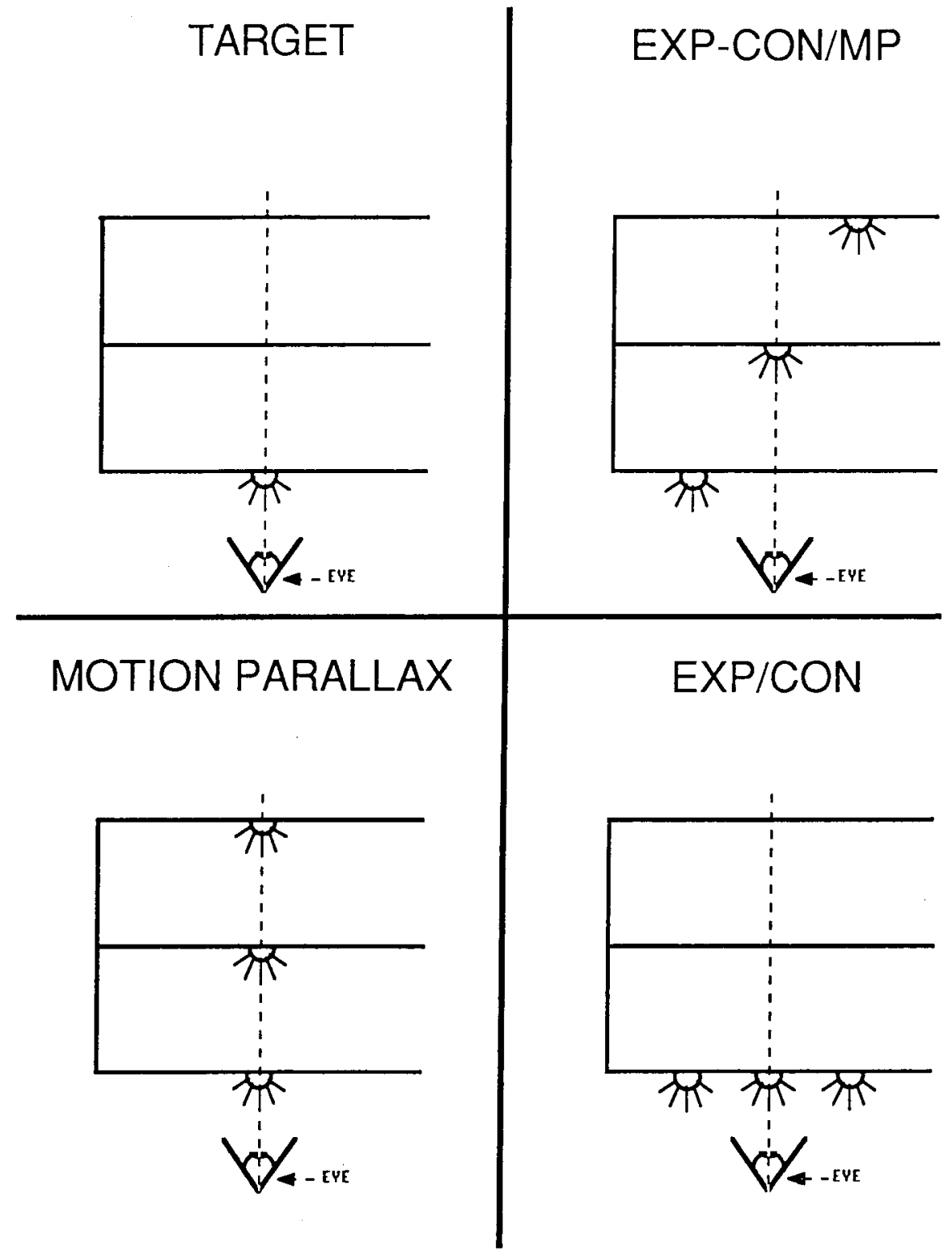

Figure 4. Diagrammatic representation of the four intermediate stimuli conditions used in the experiment, viewed from above.

potential information maximally relevant to both $\mathrm{L} / \mathrm{R}$ and F/A sway simultaneously.

Body-sway measure. A piezoelectric force plate (Kistler, Type 928A11), on which the subjects stood, measured the body's center of gravity. This was connected to a multichannel amplifier assembly (Kistler, Type 9805), which output signals specifying the center of force on the surface of the force plate. In the present experimental setting, this point can be considered an analogue of the center of gravity. ${ }^{2}$ The amplifier outputs were low-pass filtered at $10 \mathrm{~Hz}$ and digitized at $20 \mathrm{~Hz}$.

Procedure. The experiment was introduced to each subject as an investigation of visual control of quiet standing. The function of the apparatus was briefly explained and demonstrated. The subject's dominant eye was determined, using a sighting test. ${ }^{3}$ The subject stood on the force platform in stockinged feet; the stimulus array was adjusted so that the center light of the front bar was reported as being at eye level in the midsagittal plane.
The subjects stood in the standard Romberg posture for all trials, and they were told to maintain this posture during the trial but to rest and move their legs during the intertrial periods. ${ }^{4}$

The subjects were instructed to fixate the center light of each array. They were told that their only task was to maintain the Romberg posture, remain relaxed, and fixate the target. They were also instructed not to speak during the trial.

Each array was viewed once monocularly with the nondominant eye closed and patched, and once binocularly. Each condition was viewed in a random order for a block, which consisted of 12 trials (six conditions, each run binocularly and monocularly) of $30 \mathrm{sec}$ each; two blocks were run, separated by a 15 -min rest period.

Data analysis. All coordinates were specified in standard Cartesian coordinates and transformed so that the mean of all the data points for a trial was zero. Fluctuations in the center-of-force displacement were expressed as the root mean square (RMS) of the Fourier transform of the data. ${ }^{5}$ The effects of the experimental manipula- 
tions were examined with the Wilcoxon matched-pairs signed-ranked test. Nonparametrics were used, due both to the non-normal distribution of equilibrium scores and to other concerns about the appropriateness of standard parametrics; nonparametrics are common in posturography, as is indicated by the International Society of Posturography standards (Kapteyn et al., 1983). ${ }^{6}$ The Wilcoxon $t$ was converted to standard $z$ scores for ease of statistical evaluation. ${ }^{7}$

\section{Results and Discussion}

There was no significant difference between the blocks of trials, and the data were pooled for subsequent analysis. Figure 5 presents the means and standard errors of the individual conditions.

There was no systematic difference between L/R sway in binocular and in monocular fixation. F/A sway, how- ever, showed some effect in favor of binocular fixation's being more stable than monocular fixation; the effect was significant overall $(z=1.99, p<.05$; see Table 1$)$. The greater openness of F/A sway to visual influence may be due to a basement effect resulting from the biomechanics of the Romberg posture. L/R sway is restricted by the fairly rigid bone and muscle structure of the ankle, whereas the structures that control F/A sway are designed to allow movement. Therefore, the lack of binocularmonocular difference in the $L / R$ sway may be due to mechanisms that constrain L/R but not F/A sway.

The presence of a fully structured visual field resulted in less sway for both binocular and monocular fixation in both the $L / R$ and F/A dimensions of sway $(z=3.88$,
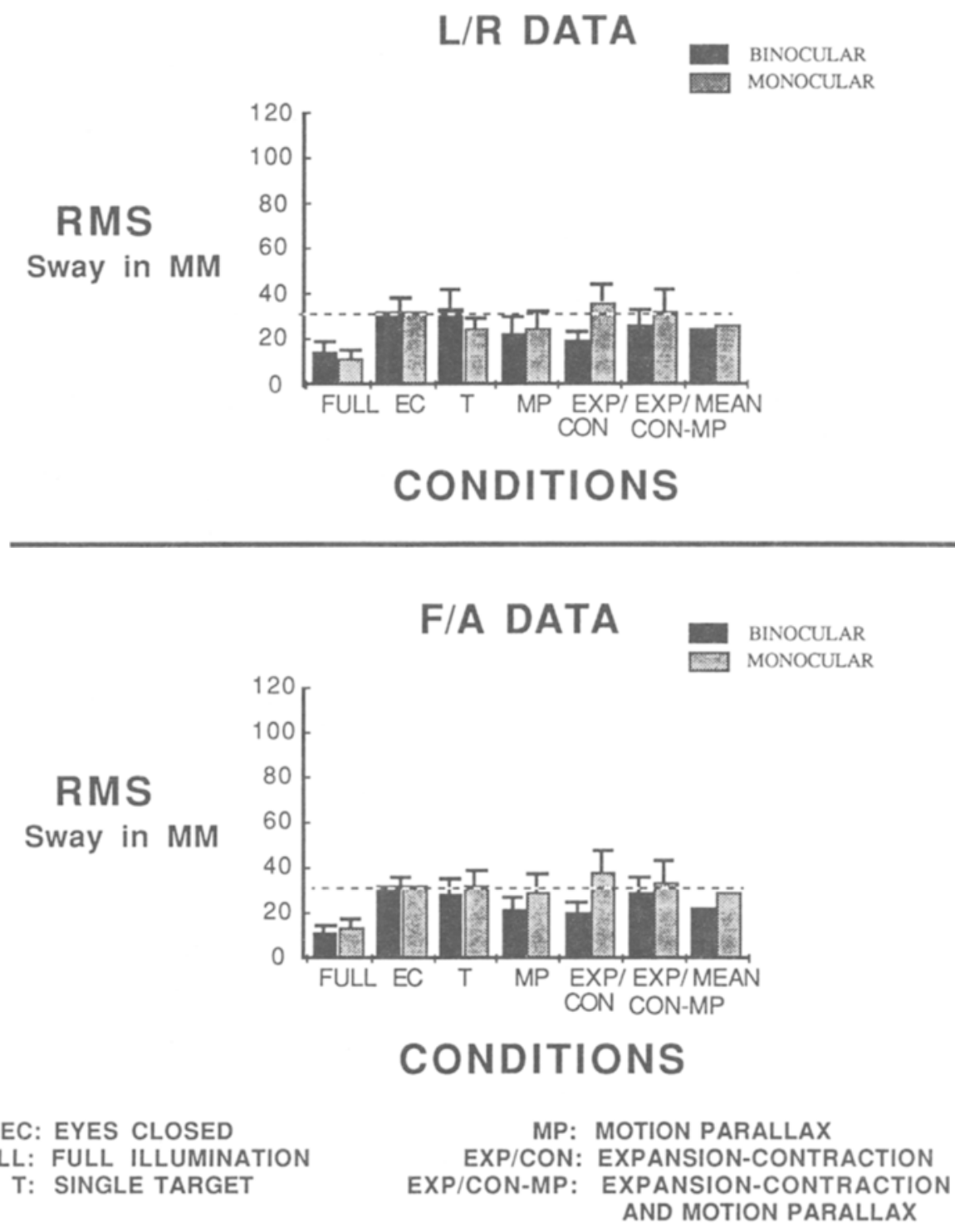

DASHED LINE INDICATES THE EYES CLOSED BASELINE

Figure 5. Means and standard errors for the L/R data (top) and F/A data (bottom) of Experiment 1. The ordinate is power as root mean square in millimeters of displacement. 
$3.40,3.92$, and 3.02 , respectively, all $p s<.01$ ). Comparison of the intermediate visual conditions (T, MP, Exp/Con, and Exp/Con-MP) to the EC baseline shows attenuation of sway, but not to a significant extent (Table 2).

Taken together, these results suggest that under the conditions of Experiment 1 the primary inputs to postural equilibrium were nonvisual. Reasonable alternatives include stabilization of equilibrium by vestibular inputs and by TPK inputs around the ankle joint, as well as passive stiffness of the ankle-joint muscles. It is quite possible that sway during quiet standing is not above threshold for the vestibular system. The absolute threshold for the vestibular system is unclear, and it is further complicated by the frequency dependence of measured threshold values (Benson, 1982). Because of these concerns Experiment 2 concentrated on TPK inputs.

\section{EXPERIMENT 2}

The results of Experiment 1 suggested that, during quiet standing in the Romberg posture, TPK (primarily from around the ankle joint) is a major source of information for the control of sway. This agrees with previous studies in which researchers have used nonrigid support surfaces (Bles, Kapteyn, Brandt, \& Arnold, 1980; Nashner, 1970, 1976, 1977; Nashner, Woollacott, \& Tuma, 1979) and reversible ischemic blocks (Diener, Dichgans, Guschlbauer, \& Mau, 1984; Elner, 1973, 1979; Elner, Gurfinkel, Lipshits, Mamasakhlisov, \& Popov, 1976; Mauritz \& Dietz, 1980). In Experiment 2, we examined postural sway under the same visual conditions as those in Experi-

Table 1

Wilcoxon $t$ (Expressed as $z$ Scores) for Experiment 1: Binocular versus Monocular Fixation of the Same Visual Field

\begin{tabular}{lcc}
\hline \multicolumn{1}{c}{ Stimulus Condition } & L/R Data & F/A Data \\
\hline Full illumination & 0.00 & 1.31 \\
Single target & -2.22 & 0.71 \\
Motion parallax & 0.15 & 0.60 \\
Expansion-contraction & 2.24 & 2.39 \\
Expansion-contraction & & \\
and motion parallax & 0.15 & 0.04 \\
Total* & 0.97 & 1.99 \\
\hline
\end{tabular}

Note-L/R = left-right body sway. F/A = fore-aft body sway. *Total indicates this comparison for all conditions combined.

Table 2

Wilcoxon $t$ (Expressed as z Scores) for Experiment 1: Experimental Conditions versus Eyes Closed

\begin{tabular}{lccccc}
\hline & \multicolumn{2}{c}{ L/R Data } & & \multicolumn{2}{c}{ F/A Data } \\
\cline { 2 - 3 } \cline { 5 - 6 } Stimulus Condition & Binocular & Monocular & & Binocular & Monocular \\
\hline Full illumination & 3.88 & 3.40 & & 3.92 & 3.02 \\
Single target & 0.60 & 1.42 & & 1.19 & 0.15 \\
Motion parallax & 1.27 & 2.05 & & 1.83 & 1.49 \\
Expansion-contraction & 1.19 & -0.22 & & 1.49 & 0.26 \\
Expansion-contraction & & & & \\
and motion parallax & 1.38 & 0.56 & & 0.45 & 0.82 \\
\hline
\end{tabular}

Note-L/R $=$ left-right body sway. F/A $=$ fore-aft body sway. ment 1 , but this time the subjects stood in a tandem (or sharpened) Romberg posture. The tandem Romberg is the same as the standard Romberg, except that the feet are placed heel to toe rather than side by side. This posture, which attenuates the usefulness of ankle TPK and reflexes, may allow visual information to have a larger influence on postural equilibrium. ${ }^{8}$ It has been successfully used in the past to examine equilibrium (e.g., Black, Wall, Rockette, \& Kitch, 1982; Fregly, 1974).

\section{Method}

Ten subjects with the same general characteristics as those of the subjects in Experiment 1 participated.

The stimulus display, conditions, body-sway measures, and data analysis were identical to those in Experiment 1. The procedure was as in Experiment 1, except that the subjects stood in a tandem Romberg posture.

\section{Results and Discussion}

There was no significant difference between the blocks of trials, and the data were pooled for subsequent analysis. Figure 6 presents the means and standard errors of the individual conditions.

Comparison of Figures 5 and 6 shows that changing from a standard to a tandem Romberg more than doubled the average sway in the EC condition (the ratios of $L / R$ and $F / A$ in Experiment 2 to $L / R$ and $F / A$ in Experiment 1 were, respectively, 2.1 and 2.5). However, the tandem Romberg had little effect in the full-binocular condition (the ratios of $\mathrm{L} / \mathrm{R}$ and $\mathrm{F} / \mathrm{A}$ in Experiment 2 to $\mathrm{L} / \mathrm{R}$ and F/A in Experiment 1 were, respectively, 1.0 and 1.6). This suggests that visual information acts by corroborating ankle-joint information, for the following reasons: In Experiment 1 (Figure 5), the local reflexes were adequate to stabilize the low-frequency sway, and the information from the long-loop visual mechanism was not involved. This can be seen in the relatively low level of overall sway; mean sway in the $\mathrm{L} / \mathrm{R}$ and F/A dimensions for binocular and monocular fixation was $24.5,26.5,23.5$, and 29.5, respectively. In Experiment 2 (Figure 6), the local postural reflexes at the ankle were inadequate to maintain equilibrium, as is evidenced by the overall greater sway; mean sway in the L/R and F/A dimensions for binocular and monocular fixation was 45.2, 55.2, 51.0, and 59.0, respectively. The increased overall sway suggests that using the tandem Romberg posture allowed the contribution of the visual information to become more evident.

The stabilizing effects of binocular as opposed to monocular fixation, which can be seen in Table 3, are largely due to the increased sway in the full-monocular condition $(z=3.88$ and 3.55 for $L / R$ and $F / A$ sway, respectively, both $p s<.01)$. This can have several possible visual causes. One source of visual information is stereopsis, which specifies, for a given object, a single 3-D spatial coordinate relative to the point of binocular fixation. This specification is based on the evaluation of (1) images projected on each retina, and (2) information about the absolute distance between the observer and object; under the present experimental conditions, binocu- 

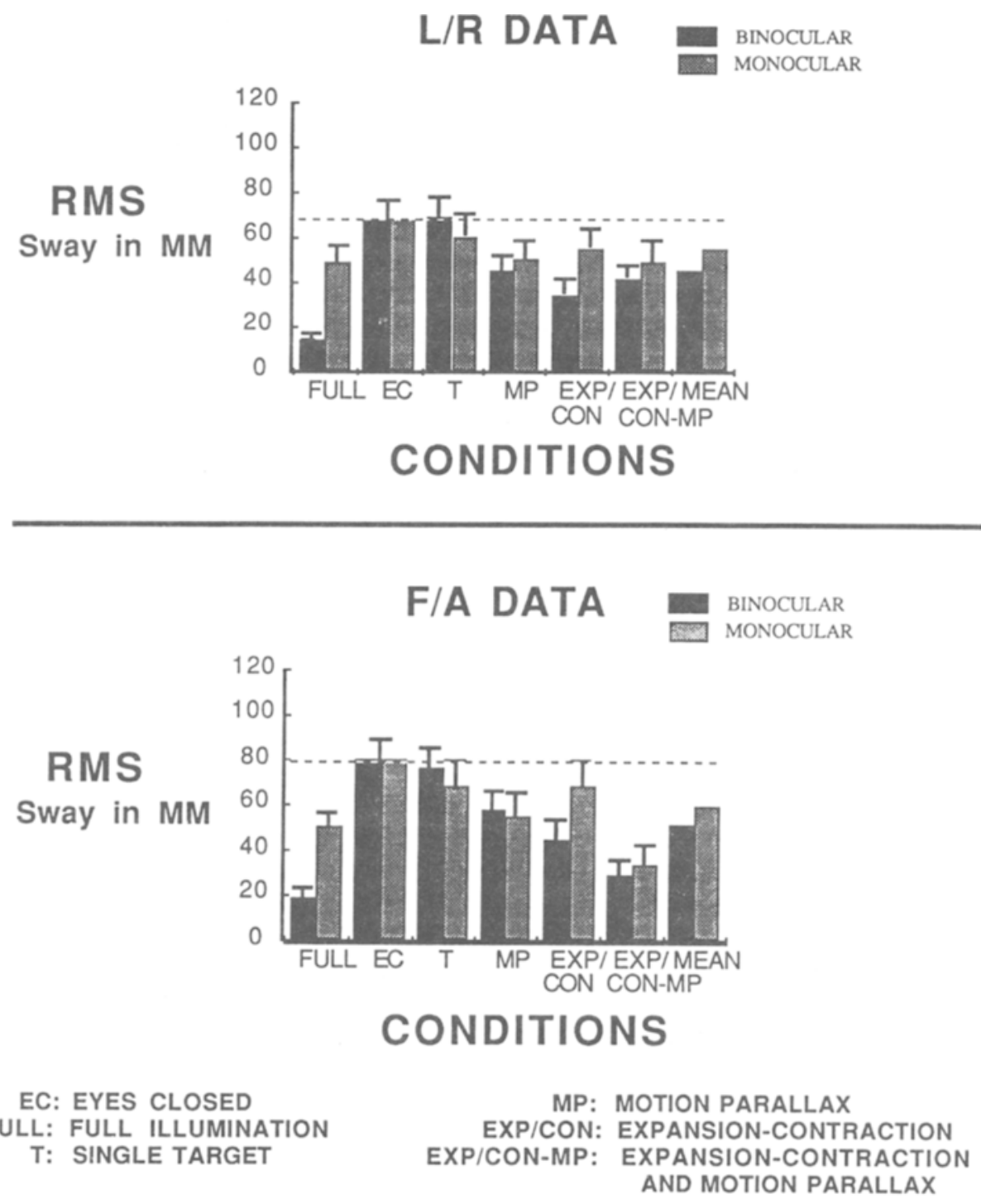

DASHED LINE INDICATES THE EYES CLOSED BASELINE

Figure 6. Means and standard errors for the L/R data (top) and F/A data (bottom) of Experiment 2. The ordinate is power as root mean square in millimeters of displacement.

lar convergence could provide such distance information. A second, yet related, source of visual information is primarily oculomotor. Work with strabismics (Mann, Hein, \& Diamond, 1979) and with normal observers whose extraocular muscles have been made less efficient through administration of systemic curare (Matin, 1982) has shown oculomotor information to be a major input for spatial localization, especially for target lights in an otherwise dark field. To the extent that visual control of postural sway is a spatial orientation task, stereopsis and oculomotor inputs may be critical sources of information for the postural control mechanism.

\section{EXPERIMENT 3}

The main findings in Experiments 1 and 2 are the differential effects of binocular and monocular fixation in favor of the former. In principle, the motion parallax information present in the target arrays should provide information equally useful to both binocular and monocular vision; however, the data show that this is not the case. This difference may be due to retinal and nonretinal factors, as described above.

To maximize the visual information available, Experiment 3 used only fully structured visual fields. This 
Table 3

Wilcoxon $t$ (Expressed as $z$ Scores) for Experiment 2: Binocular versus Monocular Fixation of the Same Visual Fields

\begin{tabular}{lcc}
\multicolumn{1}{c}{ Stimulus Condition } & L/R Data & F/A Data \\
\hline Full illumination & 3.88 & 3.55 \\
Single target & -0.97 & -1.08 \\
Motion parallax & 0.07 & -0.56 \\
Expansion-contraction & 2.01 & 1.06 \\
Expansion-contraction & & \\
$\quad$ and motion parallax & 0.30 & 0.11 \\
Total* & 2.36 & 1.19 \\
\hline
\end{tabular}

Note-L/R = left-right body sway. F/A = fore-aft body sway. *Total indicates this comparison for all conditions combined.

provided maximum retinal image structure, additional information for the evaluation of oculomotor signals (Matin \& Fox, 1989), and the most stable posture. The field contained a target that was fixated both binocularly and monocularly, in separate conditions; the data from this comparison were open to influence by both retinal and nonretinal factors. The fields used in the previous two experiments were systematically impoverished to allow systematic manipulation of the information potentially available. This level of manipulation was not needed for Experiment 3, and so, to maximize the stabilizing effects of visual fields, numerous objects were placed randomly about the room to ensure rich, full field structure, in depth $a^{2}$ well as in the frontoparallel plane.

In Experiment 3, the retinal and nonretinal factors involved in binocular and monocular fixation were separated as follows: The observers attempted to hold either the dominant eye or both eyes steady in total darkness. It was thought that this binocular-monocular comparison would be influenced primarily by nonretinal factors, because the only retinal activity would be spontaneous, random activity, which is not meaningful in terms of body sway. Comparison of a structured field with a dark field would provide further information about the influence of retinal image structure.

The final set of conditions involved closing both eyes either in total darkness or in room illumination. This manipulation permitted a measure of the effects of unstructured retinal stimulation on sway.

\section{Method}

Subjects. Eight subjects with the same general characteristics as those of the subjects in the earlier experiments participated in Experiment 3.

Stimulus display. A fixation target, consisting of a $23-\mathrm{cm}$ cross with a 9-cm diameter circle ( $1^{\circ}$ of visual angle) superimposed on it, was positioned approximately $5.2 \mathrm{~m}$ from the subject's eye. The visual array consisted of a standard laboratory environment, which provided a highly structured visual field. Illumination was identical to that in the previous experiments.

Stimulus conditions. Six stimulus conditions were used:

1. Binocular-light (BL): The subject fixated the target with both eyes open while the room was illuminated.

2. Binocular-dark (BD): The room was in complete darkness and the subject kept both eyes open.

3. Monocular-light (ML): The subject fixated the target with the dominant eye while the room was illuminated. The nondominant eye was closed and patched.
4. Monocular-dark (MD): The room was in complete darkness and the subject kept the dominant eye open. The nondominant eye was closed and patched.

5. Eyes-closed-light (ECL): The room was fully illuminated and the subject closed both eyes.

6. Eyes-closed-dark (ECD): The room was in complete darkness and the subject closed both eyes. This was used as the baseline.

Body-sway measure and data analysis. Body-sway measures and data analysis were identical to those in previous experiments.

Procedure. The procedure was similar to that used in Experiments 1 and 2, with the following changes:

The subjects stood in either the standard or the tandem Romberg posture for all trials. They were told to maintain this posture during the trial, but to rest and move their legs during the intertrial periods.

The subjects were instructed to fixate the target whenever the room lights were on, and to fixate the remembered position of the target when the room lights were off or when both eyes were closed. The subjects viewed each stimulus condition once while they were in the standard Romberg posture, and once while they were in the tandem Romberg posture. Each condition was viewed in a random order for a block, which consisted of 12 trials of $30 \mathrm{sec}$ each; two blocks were run, separated by a 15 -min rest period.

\section{Results and Discussion}

There was no significant difference between the blocks of trials, and the data were pooled for subsequent analysis. Figure 7 presents the means and standard errors.

Standard versus tandem. The standard Romberg posture is overall more stable than the tandem Romberg; this is true for most of the visual conditions (Table 4) and for all conditions combined $(z=5.31$ and 6.46 for $L / R$ and F/A sway, respectively, both $p s<.01$ ).

The comparison of the individual conditions with the ECD baseline (see Table 7) suggests that the tandem Romberg posture had the desired effect of amplifying the visual influence. However, the results are not clear, and more systematic research using different technologies will be required to separate the influence of vision, TPK, biomechanics, kinematics, and "control strategy" in the tandem Romberg posture. Some of the difficulty in interpretation may stem from differences between the visual field in the first two experiments and in the current experiment. There is clearly an effect of foot position, as has been described above, which seems to interact with visual conditions. Figure 8 indicates that, for the standard Romberg posture, changing from the motion parallax stimuli array to the full field with target in the frontoparallel plane (Experiment 1 vs. Experiment 3) primarily affected sway with monocular fixation. Conversely, sway during monocular fixation of the motion parallax array in the tandem Romberg posture is already relatively large (Experiment 2), and the change from this array to the target in the frontoparallel plane primarily affected sway with binocular fixation (Experiment 2 vs. Experiment 3). Other comparisons were nonsignificant, and therefore, for the sake of simplicity, they are not shown. There were several differences in the stimulus conditions, as has already been described (specifically, the propriospecific information and the eye-target distance). In principle, if optical/retinal explanations are adequate descriptions of the visual inputs to postural equilibrium, such differences 

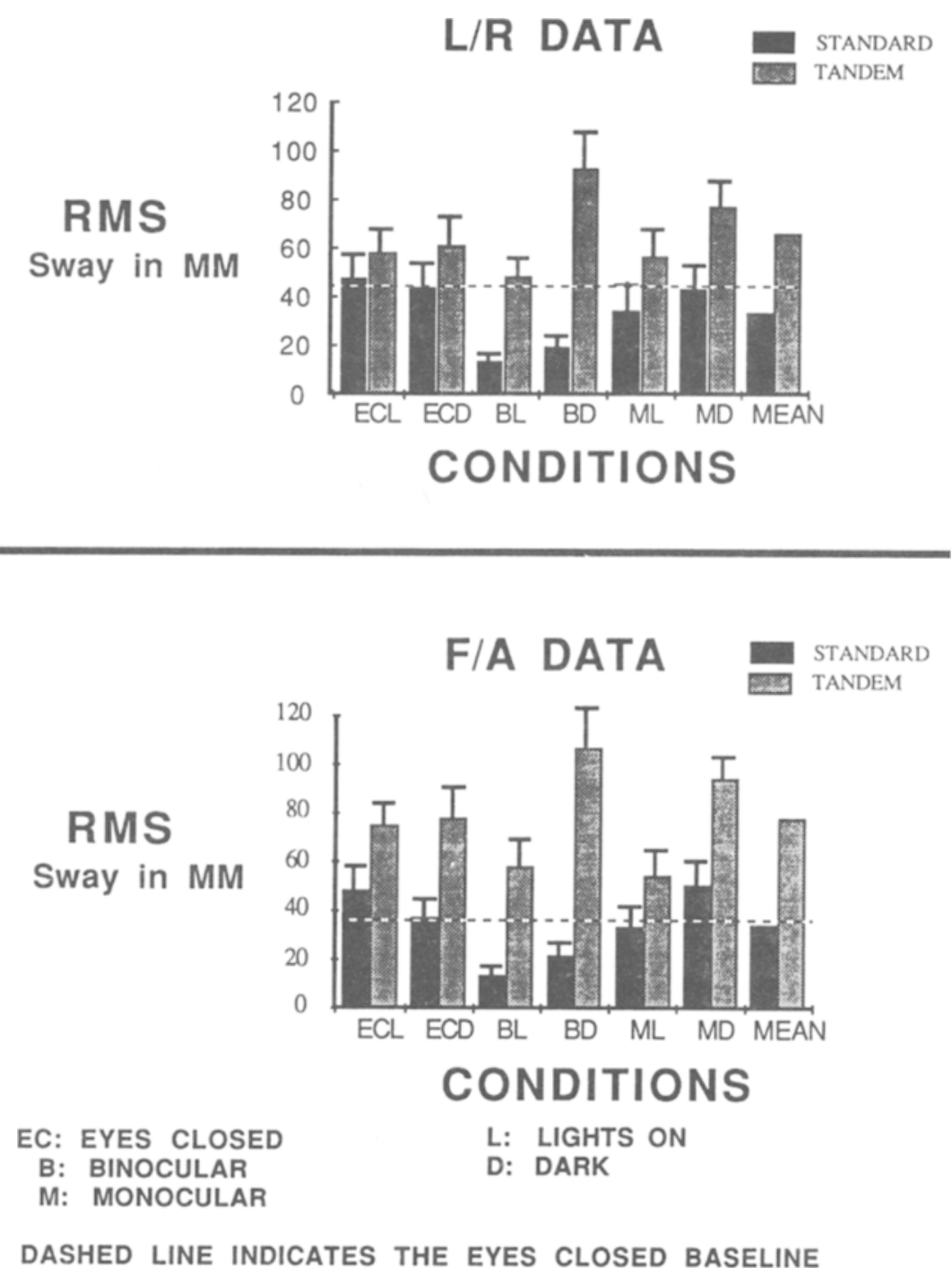

Figure 7. Means and standard errors for the $L / R$ data (top) and F/A data (bottom) of Experiment 3. The ordinate is power as root mean square in millimeters of displacement.

in the stimuli should not result in the observed differences in sway. The observed complex interaction of foot position, eye-target distance, and binocularity again suggests that simple optical/retinal explanations are insufficient to explain visual control of postural equilibrium.

Binocular versus monocular. Binocular as opposed to monocular fixation in the standard Romberg posture resulted in increased equilibrium for both F/A and L/R sway, even when the observer was in complete darkness (see Table 5, in light, $z=2.90$ and 2.95, and in dark, $z=$ 2.43 and 2.53 , for L/R and F/A sway, respectively, all $p s<.01$ ). This suggests that the stabilizing effect of binocular fixation is not mediated primarily by stereopsis or other retinal activity; if it were, there would be no difference between binocular and monocular fixation in the dark, where there appears to be no meaningful retinal stimulation (in terms of retinal displacement). Sway in the tandem Romberg posture shows no significant pattern of binocular-monocular difference (Table 5).

Room illumination. The effects of room illumination (Table 6) and related retinal stimulation indicate that field structure is an important influence. All visual field conditions showed a significant effect $(p<.05)$, except for $\mathrm{L} / \mathrm{R}$ sway in the standard Romberg posture under monocular viewing, which showed the same pattern $(p<.08)$.

Conditions versus ECD. The comparison of individual conditions to the ECD baseline (Table 7) shows that binocular fixation of a target in a structured visual field significantly stabilized sway in both the F/A and the L/R dimensions in the standard Romberg posture $(z=3.46$, both $p s<.01)$. Monocular fixation of the same target under the same conditions did not stabilize sway $(z=0.88$, $p<.72$, and $z=.24, p<.19$, respectively). This result is complicated because the exact same pattern of results 
Table 4

Wilcoxon $t$ (Expressed as $z$ Scores) for Experiment 3: Standard versus Tandem Romberg Posture during Fixation of the Same Visual Fields

\begin{tabular}{lcc}
\hline Stimulus Condition & L/R Data & F/A Data \\
\hline Eyes-closed-dark & 1.40 & 2.24 \\
Eyes-closed-light & 1.08 & 1.76 \\
Binocular-dark & 3.36 & 3.46 \\
Binocular-light & 3.46 & 3.46 \\
Monocular-dark & 2.17 & 2.48 \\
Monocular-light & 1.40 & 1.81 \\
Total* & 5.31 & 6.46 \\
\hline
\end{tabular}

Note-L/R = left-right body sway. F/A = fore-aft body sway. *Total indicates this comparison for all conditions combined.

occurred in the dark; binocular fixation of a remembered target stabilized sway $(z=2.02$ and 1.86 , for $L / R$ and F/A sway, respectively, both $p s<.05$ ), whereas monocular fixation did not $(z=0.00, p=.50$, and $z=-0.83$, $p<.20$, for L/R and F/A sway, respectively). From examination of the means of $\mathrm{BD}$ and $\mathrm{BL}$ (Figure 7), it is clear that the target and/or the field structure contributed to the stabilization. However, nonretinal factors also exerted a major influence.

The results for the tandem Romberg posture show no significant differences, but a very interesting pattern of results is present. Binocular and monocular fixation in the light, as compared with the ECD baseline, stabilizes sway; however, having the eyes open in the dark (BD and MD), compared with the ECD baseline, consistently destabilizes sway for the tandem Romberg posture. This is shown by negative $z$ scores with probabilities of approximately 0.1 for all four $z$ comparisons. This pattern raises the question of why total darkness should be evaluated at all.

Eye closure versus darkness. The difference between eye closure and darkness has not been reported previously. ${ }^{9}$ However, comparison of visual stabilization and eye closure during postural perturbation suggests that eye closure is not equivalent to the minimization of visual motion cues (Nashner \& Berthoz, 1978; Soechting \& Berthoz, 1979; Vidal, Berthoz, \& Millanovoye, 1982). "Stabilizing" the visual field relative to body sway alters the feedback relation between body motion and transformation or displacement of the retinal image; postural sway increases with a stabilized field, due to an inappropriate dependence upon this altered "reafference" (to use traditional terminology). When the eyes are closed, there is no "expected" relation between body sway and change in the visual field, and so the same dependency or influence is not present as when the eyes are open. In brief, there is the possibility that eye closure results in visual information's being evaluated in a manner different from how it is evaluated when the eyes are open. The difference cannot be precisely specified at this point. ${ }^{10}$ The same logic applies to the present experiment, in that it also altered the normal relation between body sway and visual field transform.

Work on height vertigo (Bles et al., 1980) demonstrates that the unstructured or "empty" visual field associated with conditions inducing physiological height vertigo also induces postural disequilibrium. ${ }^{11}$ This effect is most likely due to a mechanism that modulates vestibular and/or vestibulospinal mechanisms relative to visual inputs. Such a mechanism has been demonstrated for the linear vestibulo-ocular reflex, where the state of vergence has been found to be the most important single variable underlying the phenomenon (Paige, 1989).

\section{SUMMARY OF MAIN RESULTS}

1. A fully illuminated and structured visual field results in maximal postural equilibrium.

2. With normal ankle-joint information, as in the standard Romberg posture, minimal visual field structure (the

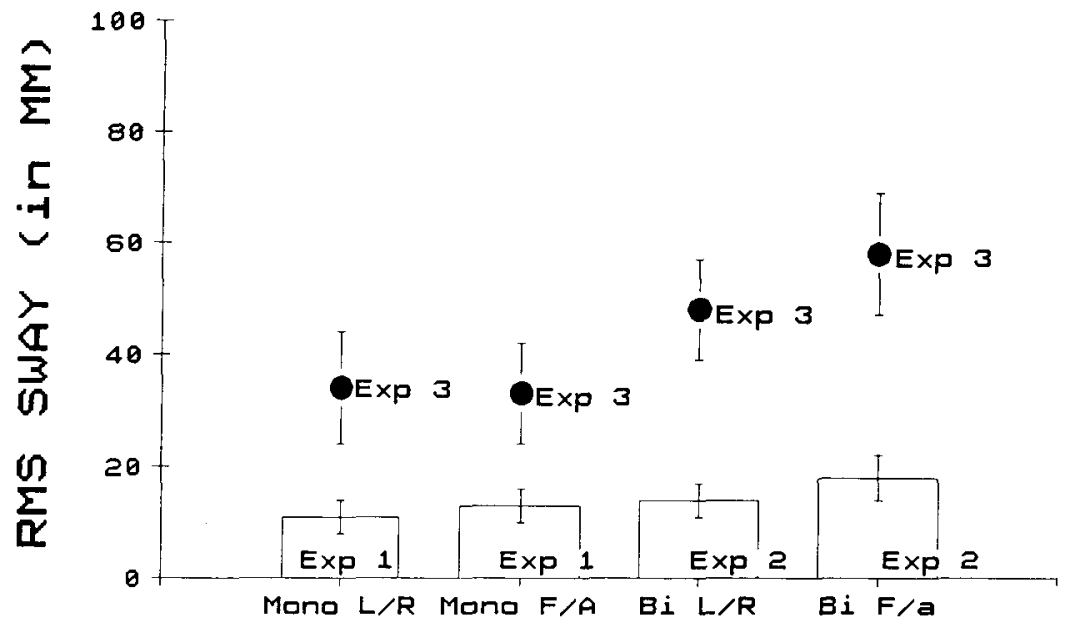

Figure 8. Means from Experiments 1 and 3 and Experiments 2 and 3, illustrating the effects of the different visual stimuli. The ordinate is power as root mean square in millimeters of displacement. 
Table 5

Wilcoxon $t$ (Expressed as $z$ Scores) for Experiment 3: Binocular versus Monocular Fixation of the Same Visual Fields

\begin{tabular}{cccccc}
\hline \multirow{2}{*}{ Stimulus } & \multicolumn{2}{c}{ L/R Data } & & \multicolumn{2}{c}{ F/A Data } \\
\cline { 2 - 3 } \cline { 5 - 6 } Condition & Standard & Tandem & & Standard & Tandem \\
\hline Light & 2.90 & 0.16 & & 2.95 & -0.47 \\
Dark & 2.43 & -1.45 & & 2.53 & -0.46 \\
\hline
\end{tabular}

Note-L/R = left-right body sway. F/A $=$ fore-aft body sway.

Table 6

Wilcoxon $t$ (Expressed as $z$ Scores) for Experiment 3: Full Illumination versus Complete Darkness during Fixation of the Same Visual Fields

\begin{tabular}{lccccc}
\hline \multirow{2}{*}{$\begin{array}{l}\text { Stimulus } \\
\text { Condition }\end{array}$} & \multicolumn{2}{c}{ L/R Data } & & \multicolumn{2}{c}{ F/A Data } \\
\cline { 2 - 3 } \cline { 5 - 6 } \cline { 5 - 6 } & Standard & Tandem & & Standard & Tandem \\
\hline Eyes closed & -0.36 & -0.36 & & -0.67 & -0.36 \\
Binocular & 2.07 & 2.11 & & 1.96 & 1.96 \\
Monocular & 1.40 & 1.86 & & 2.02 & 2.53 \\
\hline
\end{tabular}

Note-L/R = left-right body sway. F/A = fore-aft body sway.

Table 7

Wilcoxon $t$ (Expressed as z Scores) for Experiment 3: Experimental Conditions versus Eyes Closed

\begin{tabular}{lccccc}
\hline & \multicolumn{2}{c}{ L/R Data } & & \multicolumn{2}{c}{ F/A Data } \\
\cline { 2 - 3 } \cline { 5 - 6 } Stimulus Condition & Standard & Tandem & & Standard & Tandem \\
\hline Binocular-light & 3.46 & 0.93 & & 3.46 & 1.14 \\
Binocular-dark & 2.02 & -1.40 & & 1.86 & -1.45 \\
Monocular-light & 0.88 & 0.36 & & 0.72 & 1.24 \\
Monocular-dark & 0.00 & -1.24 & & -0.83 & -1.29 \\
\hline
\end{tabular}

Note-L/R $=$ left-right body sway. F/A = fore-aft body sway.

four LED conditions) results in no significant stabilization of postural sway. This suggests a strong ankle-joint input to equilibrium.

3. Altering ankle-joint information by using a tandem Romberg posture leads to minimal field structure (the four LED conditions) stabilizing postural sway. This suggests a long loop mechanism involving visual modulation of postural reflexes.

4. Binocular fixation stabilizes sway more than does monocular fixation of the same visual field, even though the field contains information that is equally useful to both. This suggests that the visual influence involves more than just optical information.

5. The binocular-monocular difference remains, even when the visual field is totally dark. This suggests that the underlying mechanism is not stereopsis.

6. Altering ankle-joint information by using the tandem Romberg posture and keeping one or both eyes open in the dark (rather than eyes closed) destabilizes sway. This indicates a complex mechanism that selectively evaluates sensory input on the basis of expected patterns of sensation.

\section{GENERAL DISCUSSION}

Postural equilibrium during quiet standing is a complex sensorimotor task. In human beings, it involves maintaining the body's COG within the area of a fixed base of support. Traditionally, this visual input has been analyzed in terms of optical and retinal phenomena, but the data presented here cannot be explained adequately in these terms, and they suggest that nonretinal factors are also involved.

As a first approximation, postural equilibrium can be considered an egocentric spatial orientation task, which involves ongoing monitoring of the position of an object (the body) relative to some spatial coordinate system. For this visual task, the locus of retinal stimulation acquires significance only if it is defined in relation to an independent frame of reference that specifies location and orientation of the retinal surface. Recently, an expanded comparator mechanism that allows information about eye position from both extraretinal and retinal sources has been proposed (Fox, 1986; Matin \& Fox, 1989; Petito, Greenwald, \& Fox, 1988). This mechanism suggests that in the presence of a highly structured visual field, spatial localization is primarily influenced by the main lines of organization of the visual field (visual-field-referenced eye-position information); when such a field is not present (e.g., in darkness), spatial localization is primarily influenced by a body-referenced mechanism involving a retinal signal and an independent reference signal that specify head position and eye position relative to the head (bodyreferenced eye-position information). This mechanism suggests that in the presence of a highly structured field, postural sway is primarily influenced by the moving contours of the field; however, the importance of nonretinal factors (specifically, oculomotor information) is implied in many experiments, including the present ones (Edwards, 1942, 1946; Gantchev, 1980; Iwase et al., 1979; Miyoshi, 1979; Miyoshi, Shirato, \& Hiwatashi, 1979; Oblack, Mihelin, \& Gregoric, 1976; Paulus et al., 1984; Taguchi, 1979; Tokumasu, Tashiro, \& Yoneda, 1979; White et al., 1980).

The major findings in the present experiments comprise (1) the effects of highly structured, whole visual fields, (2) the effects of the target arrays, (3) the difference between binocular and monocular fixation, and (4) the effects of involuntary elimination of visual field structure (eyes open in the dark). Each can be explained within the framework offered here.

It has been suggested (Dichgans \& Brandt, 1978) that the central $30^{\circ}$ is related to control of eye movement, but that the peripheral field is related to the sense of body motion and equilibrium. In the present experiments, full field structure provided both central and peripheral structure and attenuated sway. The field was stationary and provided maximal feedback resulting in minimal postural sway. However, the central/peripheral distinction is not absolute (Andersen \& Braunstein, 1985). The target arrays in Experiments 1 and 2 were limited to the central $30^{\circ}$ of an otherwise dark field; there was no peripheral field structure. These arrays, compared with the eyesclosed condition, attenuated sway, but not to the extent that full visual fields did. This suggests that the visual influence occurred through an alternate mode of control 
such as body-referenced eye-position information. This mode adds information from the visual system to vestibular and TPK inputs. The addition of information from the visual system will, because of the visual system's high precision, attenuate sway relative to eyes-closed conditions, but not to the extent that a full, structured, stable field would.

A consistent finding in the present experiments is that binocular fixation, as compared with monocular fixation of the same visual array, attenuated sway; this is a problem for both the visual flow and the comparator theories. Information in the visual flow field is, potentially, equally useful to both monocular and binocular vision. Traditional comparator theory cannot explain this difference, unless independent reference signals for each eye are postulated.

That there are independent reference signals for each eye is suggested by experiments done on uniocular prism adaptation (Mann et al., 1979) in strabismics, spatial localization after uniocular strabismic surgery (Matin, 1982), and noninvasive therapy for strabismics (Petito et al., 1988). The data from these experiments suggest that each of the two eyes has separate reference signals for its position in space. The independence of these signals is not evident in normal observers, but this does not rule out independent reference signals for each eye. In normals, the noise in the two signals would be minimized by their high correlation, perhaps through a statistical "common mode noise rejection" type of filtering; and a single reference signal is a reasonable, although not necessarily complete, description.

The last finding to be considered here consists of the data from keeping the eye open in the dark (Experiment 3). Both eyes closed and eyes open in the dark decouple visual feedback from body sway, but they have different effects on sway control. In the standard Romberg posture, the pattern of results is unclear. Binocular fixation in the dark of a remembered target attenuated sway relative to eye closure, whereas monocular fixation did not. Caution must be used in interpreting this result, because the data (Experiment 1 vs. Experiment 2) suggest that sway in the standard Romberg posture was less influenced by vision than sway in the tandem Romberg posture was. The results from observers in the tandem Romberg posture show a clearer difference for eyes open in the dark, where sway is less stable than it is when the eyes are closed. This difference, though it is not statistically significant, is very clear.

The eyes-closed condition can be considered an elimination of the visual influence on postural sway. Eyes open in the dark maintains the visual control loop, but no useful information is available. In essence, the visual system compensates for its own actions, but external actions are not compensated for in the same manner. We can consider this within the framework of the "effort of the will," which was proposed by Helmholtz (1866/1962) to account for the stability of the visual world. When the eyes are open, there is an "expectation" of a pattern of visual stimulation, and the absence of such a pattern results in incongruence; with the eyes closed, there is no expectation. This may also account for the results of experiments on individuals with clinical or experimentally produced visual acuity defects. Such observers show no measurable visual stabilization of postural sway (in fact, they exhibit increased sway in some cases) relative to sway with eyes closed (Brandt, Esser, Buchele, \& Krafczyk, 1982; Edwards, 1942, 1946; Paulus et al., 1984). Similar reasoning has been used to explain posturographic results during visual conditions that induce physiological height vertigo (Bles et al., 1980).

An analogous situation in which visual information is evaluated uniquely is saccadic suppression. During a saccade, vision is severely impaired, but this impairment is not perceived by the subject. This suppression occurs 30-40 msec before the saccade starts and lasts for $100-120 \mathrm{msec}$; comparable interruptions (100 msec) of sight by a shutter are readily perceived. This suggests that the visual system compensates for its own actions, but that interruptions by external means are not compensated for in the same manner. Saccadic suppression and suppression during eye blinks can be considered a "shutting off" of the normal mode of processing visual information. The relevance is that in Experiment 3, the eyes were open and information was potentially available from the visual system; however, retinal stimulation was interrupted by external means and this was not compensated for by the visual system. That eyes open in the dark can change the processing of visual information can be seen by the response of cells in the lateral geniculate nuclei (LGN). The LGN has been suggested as the site of a comparator that distinguishes object motion from eye movement. This visual comparator is based on the response of $S$ and $T$ cells in the LGN: $S$ cells have a sustained firing pattern, which is related to instantaneous eye position; $T$ cells have transient firing patterns, which are related to saccades and other eye movements. Neither response has been observed to occur readily in the dark, which suggests that this mechanism functions differently when the observer's eyes are open in the dark (Carpenter, 1977). Similar mechanisms may be involved in the postural control system when the eyes are open in the dark; the visual system is linked into the postural control system, but the feedback information that should be available is interrupted by external means or is not available.

These results indicate that the visual influence on postural equilibrium results from a complex synergy that modulates postural reflexes. A simple optical/retinal explanation is not sufficient. Visual control of posture might be profitably explored in the more general framework of visual spatial localization, along with other areas of visually guided, spatially oriented behavior.

\section{REFERENCES}

Andersen, G. J., \& Braunstein, M. L. (1985). Induced self-motion in central vision. Journal of Experimental Psychology: Human Perception \& Performance, 11, 122-132.

amblaro, B., Carblanc, A., \& Cremieux, J. (1980). Position versus 
visual motion cues in human body sway. Paper presented at Symposium on the Study of Motion Perception. The Netherlands.

Amblard, B., \& CremieUX, J. (1976). Role de l'information visuelle du mouvement dans le maintien de l'équilibre postural chez l'homme. Agressologie, 17(C), 25-36.

Bendat, J. S., \& Piersol, A. G. (1971). Random data: Analysis and measurement procedures. New York: Wiley-Interscience.

Benson, A. J. (1982). The vestibular sensory system. In H. B. Barlow \& J. D. Mollon (Eds.), The senses (pp. 333-368). Cambridge, England: Cambridge University Press.

Berthoz, A., Lacour, M., Soechting, J. F., \& Vidal, P. P. (1979). The role of vision in the control of posture during linear motion. In R. Granit \& O. Pompeiano (Eds.), Reflex control of posture and movement: Progress in brain research V5O (pp. 197-209). Amsterdam: Elsevier/North-Holland Biomedical Press.

Black, F. O., Wall, C., Rockette, H. E., \& KItCh, R. (1982). Normal subjects' postural sway during the Romberg test. American Joumal of Otolaryngology, 3, 309-318.

Bles, W., Kapteyn, T. S., Brandt, T., \& Arnold, F. (1980). The mechanism of physiological height vertigo: II. Posturography. Acta Otolaryngology, 89, 534-540.

Brandt, T., Esser, J., Buchele, W., KrafczyK, S. (1982). "Visuospinal ataxia" caused by disorders of eye movements. In A. Roucoux \& M. Crommelinck (Eds.), Physiological and pathological aspects of eye movements (pp. 425-430). The Hague: W. Junk.

Carpenter, R. H. S. (1977). Movements of the eyes. London: Pion. Dichgans, J., BrandT, T. (1978). Visual-vestibular interaction: Effect on self-motion perception and postural control. In R. Held, H. W. Leibowitz, \& H. L. Teuber (Eds.), Handbook of sensory physiology (Vol. 8). Berlin: Springer-Verlag.

Dichgans, J., Held, R., Young, L. R., \& Brandt, T. (1978). Moving visual scenes influence the apparent direction of gravity. Science, 178, 1217-1219.

Dichgans, J., Mauritz, K. H., Allum, J. H. J., \& Brandt, T. (1975). Postural sway in normal and ataxic patients: Analysis of the stabilizing and destabilizing effects of vision. Agressologie, 17(C), 15-24.

Diener, H. C., Dichgans, J., Guschlbauer, B., \& Bacher, M. (1986). Role of visual and static influences on dynamic posture control. Human Neurobiology, 5, 105-113.

Diener, H. C., Dichgans, J., Guschlbauer, B., \& MaU, H. (1984) The significance of proprioception on postural stabilization as assessed by ischemia. Experimental Brain Research, 56, 103-109.

Droulez, J., Berthoz, A., VIDAL, P. P. (1985). Use and limits of visual-vestibular interaction in the control of posture. In $\mathrm{M}$. Igarashi \& F. O. Black (Eds.), Vestibular and visual control of posture and locomotor equilibrium (pp. 14-21). Basel: Karger.

EDWARDs, A. S. (1942). The measurement of static ataxia. American Journal of Psychology, 55, 171-188.

EDWARDS, A. S. (1946). Body sway and vision. Journal of Experimental Psychology, 36, 526-535.

ELNER, A. M. (1973). Possibilities of correcting the urgent voluntary movements and the associated postural activity of human muscles. Biophysics, 18, 966-971.

ELNER, A. M. (1979). The role of proprioception in the control of postural muscular activity in man. Agressologie, 20(B), 131-132.

ElNer, A. M., GuRfinkel, V. S., LipShits, M. I., MAMASAKhlisov, G. V., \& Popov, K. E. (1976). Facilitation of stretch reflex by additional support during quiet stance. Agressologie, 17(A), 15-20.

Fox, C. R. (1986). Some visual influences on human postural equilibrium. Dissertation Abstracts International, 47, 2652B. (University Microfilms No. ADG86-17008, 8612)

FreGLY, A. R. (1974). Vestibular ataxia and its measurement in man. In H. H. Komhuber (Ed.), Handbook of sensory physiology (Vol. 6/2, pp. 321-360). Berlin: Springer-Verlag.

Gantchev, G. N. (1980). Studies on postural activity in man. Agressologie, 21(C), 35-40.

GiBson, J. J. (1950). The perception of the visual world. Boston: Houghton Mifflin.

GiBson, J. J. (1966). The senses considered as perceptual systems. Boston: Houghton Mifflin.

GiBSON, J. J. (1979). The ecological approach to visual perception. Boston: Houghton Mifflin.
HeLmholtz, H. voN. (1962). Treatise on physiological optics (Vol. 3; J. P. C. Southall, Trans.). New York: Dover. (Original work published 1866)

Holst, E. von, \& Mittelstaedt, H. (1973). The reafference principle. In Robert Martin (Trans.), The behavioral physiology of animals and man (pp. 139-172). Coral Gables, FL: University of Miami Press. (Original work published 1950)

Iwase, Y., Uchida, T., Hashimoto, M., SuZuki, N., Takegami, T., \& Yамамото, Y. (1979). The effect of eye movements on the upright standing in man. Agressologie, 20(C), 193-194.

JoHANSSON, G. (1976). Spatial temporal differentiation and integration in visual motor perception. Psychology Review, 30, 379-393.

Kapteyn, T. S., Bles, W., Nuokiktuen, C. J., Kodde, L., Nasses, C. H., MoL, J. M. F. (1983). Standardization in platform stabilometry being a part of posturography. Agressologie, 24(7), 321-326.

LEE, D. N., \& LISHMAN, J. R. (1975). Visual proprioceptive control of stance. Journal of Human Movement Studies, 7, 87-95.

LEE, D. N., Lishman, J. R. (1977). Vision-the most effective source of proprioceptive information for balance control. Agressologie, 18(A), 83-94.

LeiBowitz, H. W., OWENS, D. A. (1975). Anomalous myopia and the intermediate dark focus of accommodation. Science, 189, 646-648.

Lestienne, F., Soechting, J., \& Berthoz, A. (1977). Postural readjustments induced by linear motion of visual scenes. Experimental Brain Research, 28, 363-384.

Lishman, J. R., LeE, D. N. (1973). The autonomy of visual kinesthesis. Perception, 2, 287-294.

Mann, V. A., Hein, A., Dinmond, R. (1979). Patterns of interocular transfer of visuomotor coordination reveal differences in the representation of visual space. Perception \& Psychophysics, 25, 35-41.

MAtin, L. (1982). Visual localization and eye movements. In A. H. Wertheim, W. A. Wagenaar, \& H. W. Leibowitz (Eds.), Tutorials on motion perception (pp. 000-000). New York: Plenum.

MAtin, L., Fox, C. R. (1989). Visually perceived eye level and perceived elevation of objects: Linearly additive influences from visual field pitch and from gravity. Vision Research, 29, 315-324.

Mauritz, K. H., \& DiETz, V. (1980). Characteristics of postural instability induced by ischemic blocking of leg afferents. Experimental Brain Research, 38, 117-119.

Miroshi, T. (1979). Vestibular and optokinetic disequilibrium. Agressologie, 20(C), 199-200.

Miroshi, T., Shirato, M., \& Hiwatashi, S. (1979). Two phasic body sway by optokinetic stimulation. Agressologie, 20(C), 119-125.

NASHNER, L. M. (1970). Sensory feedback in human posture control (Tech. Rep. No. MVT 70-3). Boston: Massachusetts Institute of Technology.

NASHNER, L. M. (1976). Adapting reflexes controlling the human posture. Experimental Brain Research, 26, 59-72.

NASHNER, L. M. (1977). Fixed patterns of rapid postural responses among leg muscles during stance. Experimental Brain Research, 30 , 13-24.

NAShNer, L. M., \& BerThOz, A. (1978). Visual contribution to rapid motor responses during postural control. Brain Research, 150, 403-407.

Nashner, L. M., Woollacott, M., \& Tuma, G. (1979). Organization of rapid responses to postural and locomotor-like perturbations of standing man. Experimental Brain Research, 36, 463-476.

Oblack, B., Mihelin, M., \& Gregoric, M. (1976). Computer analysis of the correlation between visual stimulation, ocular movements and stabilograms. Agressologie, 17(C), 11-14.

OWENS, D. A., \& LeIBowitz, H. W. (1983). Perceptual and motor consequences of tonic vergence. In C. M. Schor \& K. J. Cuiffreda (Eds.), Vergence eye movements: Basic and clinical aspects (pp. 000-000). Woburn, MA: Butterworth.

PAIGE, G. D. (1989). The influence of target distance on eye movement responses during vertical linear motion. Experimental Brain Research, 77, 585-593.

Paulus, W: M., Straube, A., \& Brandt, T. (1984). Visual stabilization of posture. Brain, 107, 1143-1163.

Petito, G. T., Greenwald, I., \& Fox, C. R. (1988). A model of spatial localization and its application to strabismus. American Journal of Optometry \& Physiological Optics, 65, 108-117. 
Soechting, J. F., \& Berthoz, A. (1979). Dynamic role of vision in the control of posture in man. Experimental Brain Research, 36, $551-561$.

TAGUCHI, K. (1979). Effects of optokinetic stimulation on the center of gravity during normal standing. Agressologie, 20(C), 197-198.

Tokumasu, K., TASHIRo, N., \&oneda, S. (1979). Visual influences on body stability in Romberg's posture. Agressologie, 20(C), 187-189.

Vidal, P. P., Berthoz, A., Millanovoye, M. (1982). Difference between eye closure and visual stabilization in the control of posture in man. Aviation, Space, \& Environmental Medicine, 53, 166-170.

WERTHEIM, A. H., \& BLES, W. (1984). A re-evaluation of cancellation theory: Visual, vestibular and oculomotor contributions to perceived object motion (Report No. IZF 1984-88). Soesterberg, The Netherlands: TNO Institute for Perception.

White, K. D., Post, R. B., \& Leibowitz, H. W. (1980). Saccadic eye movements and body sway. Science, 208, 621-623.

White, K. D., Woods, C. B., \& Post, R. B. (1989). Eyelid closure changes postural stability and vestibulo-ocular reflexes. Investigative Ophthalmology \& Visual Science, 30, 182.

Woods, C. B., \& WHTE, K. D. (1989). Visual-vestibular integration without moving retinal images. Investigative Ophthalmology \& Visual Science, 30, 182.

\section{NOTES}

1. It is likely that each of the stimulus displays provided more information than that which is listed. The list specifies which dimension of sway the potential information is maximally relevant to.

2. Several researchers treat the center of force as a direct projection of the COG. If the movement is slow and certain other conditions are met, this is reasonable. However, the distinction between the centers of force applied to the measuring system and the COG of the body should be maintained until the appropriate proofs and standards are developed.

3. Holding it at arm's length, the subject aligned a pencil with a line approximately $4 \mathrm{~m}$ away. Each eye was alternately covered, and the subject reported relative movement of the pencil and line.

4. In the Romberg posture, the subject stands with feet together, knees straight, and torso and head erect, arms folded over chest. This posture was chosen because of its use as a standard in neurological examinations, so that the present data would be consistent with other published data.

5. To obtain zero mean data, the DC offset was calculated as the mean value of the time history. This mean value was subtracted from each data point. RMS was obtained by summing the square roots of the discrete power spectral estimates (obtained from the Fourier transform of the data), which were scaled such that a sinusoidal waveform of peak $A$ would have a corresponding spectral peak magnitude $A^{2}$. A full discussion of the logic and the mathematics used for this data transform can be found in Bendat and Piersol (1971).

6. The classic parametric methods are derived with explicit assumptions about population distributions and parameters. Order techniques depend directly on elementary probability theory, and they are comparatively free from assumptions about population distributions and parameters. However, both can be considered equivalent, in the following sense: Analysis of variance is based on the assumption of equal means, which imply, and are implied by, the identical population hypothesis. The Wilcoxon is based on the equal likelihood hypothesis, which is implied by the identical population hypothesis. If the equal likelihood hypothesis is rejected, the identical population hypothesis can also be rejected.

In the context of the present experiments, the Wilcoxon is preferred; little is known about the underlying distributions, but what is known suggests that they are skewed rather than normal. Independent concerns about the appropriate measurement scale also suggest that parametric statistics may not be appropriate for posturography.

7. The appropriate probability level to be used is a matter of custom for single-hypothesis testing, a matter of controversy for multiplehypothesis testing. So, while acknowledging the concerns arising from multiple hypothesis testing, the customary $p<.05$ and $p<.01$ levels are used for semantic simplicity. The $z$ score is also reported to allow the reader to apply more conservative statistical standards if desired.

8. It should be acknowledged that normal postural reflexes may be less efficient in the tandem Romberg posture, and that the kinesiology of postural corrections may be different. Furthermore, because the base of support for F/A sway is doubled and the L/R base of support is halved, the relation between the two may be altered.

9. Two recent conference abstracts (White, Woods, \& Post, 1989; Woods \& White, 1989) have reported replication of a difference between eyes open and closed in the dark. Specifically, when subjects stood on one leg in the dark with their eyes closed, their head movement was greater than it was when their eyes were open. These differences are opposite in direction to the ones reported here; this may be due to methodological differences. The key point is that an independent laboratory, using radically different techniques, has confirmed the finding that eye closure is not merely the equivalent of zero retinal image.

10. In linear systems theory, this can be expressed in the following manner: Closing the eyes eliminates the possibility of a retinal pattern, indicating zero error in a negative feedback loop; the error signal will be determined by the random activity of the retina. It is unclear whether or not linear systems analysis is an appropriate level of analysis for the postural control system (Droulez, Berthoz, \& Vidal, 1985). Until this issue is resolved, the conceptual analysis presented in the present paper is to be preferred.

11. That the visual system treats both empty and dark fields in a similar manner is indicated by work on "dark focus" and "dark vergence" (Leibowitz \& Owens, 1975; Owens \& Leibowitz, 1983). These phenomena indicate that without adequate visual stimuli, the eyes assume a posture that is intermediate in the functional ranges of accommodation and binocular vergence. This posture, which is assumed when the field is dark or minimally structured, may be involved in the data presented here.

(Manuscript received January 4, 1988; revision accepted for publication November $20,1989$. 